\title{
TOPOGRAPHIC MAPPING AND ANALYSIS BASED ON 3D RECONSTRUCTION MODEL OF SIMULATED ASTEROID
}

\author{
R. Wang ${ }^{1}$, K. Di ${ }^{1}$, W. Wan ${ }^{1}$, Z. Liu ${ }^{1, *}$, Y. Wang ${ }^{1}$, W. Liang ${ }^{1}$, Y. Wang ${ }^{2}$, X. Chen ${ }^{2}$, S. Zhi ${ }^{2}$ \\ ${ }^{1}$ State Key Laboratory of Remote Sensing Science, Aerospace Information Research Institute, Chinese Academy of Sciences, \\ Beijing, China - (wangrz, dikc, wanwh, liuzq, wangyx716)@radi.ac.cn, liangwuyang1026@163.com \\ ${ }^{2}$ Innovation academy for microsatellites of CAS, Chinese Academy of Sciences, Shanghai, China - wangyam09@163.com, \\ xinchen8905@163.com, zhishuai0705@163.com
}

Commission III, ICWG III/II

KEY WORDS: 3D Reconstruction, Simulated Asteroid, DEM, Topographic Mapping, Topographic Analysis, SfM

\begin{abstract}
:
Asteroid exploration is of great scientific significance in understanding the evolution of the solar system. Unlike lunar missions, asteroids are far away from the earth, the communication between the spacecraft and the ground station has a large delay. Moreover, the small size, irregular shape and uneven mass distribution of asteroids further increase the difficulty of landing missions. This paper presented a topographic mapping and analysis method based on three-dimensional (3D) reconstruction model of a simulated asteroid. First, 3D reconstruction of a simulated asteroid was achieved using Structure from Motion (SfM) technique. Then Digital Elevation Model (DEM) and Digital Orthophoto Map (DOM) were produced based on the 3D point cloud data. By analysing the DEM data, we finally obtained the obstacles distribution map. In order to verify the proposed method, we used a simulated asteroid to conduct experiments. And the experimental results demonstrated the effectiveness of the developed method. The topographic mapping and analysis method presented in this study can be applied to landing/sampling area selection in further asteroid exploration research.
\end{abstract}

\section{INTRODUCTION}

Deep space exploration is the main way for human beings to explore the universe, which is of great significance to the development of human civilization. With the rapid development of space science and technology, scientists began to explore asteroids in addition to the Moon, Mars, and other planets in the solar system. Asteroid exploration is significant to understanding the evolution of the solar system. Many countries around the world began to carry out asteroid exploration activities and develop long-term exploration plans (Wu et al., 2013). The asteroid exploration mission is of great scientific significance. Asteroids are rich in available resources, including high-value metals and minerals. At the same time, new technologies developed in Pin-Point Landing and sampling return missions can also lay the foundation for future space exploration, promoting the progress of key technologies (Johnson and Montgomery, 2008).

There are many difficulties in asteroid exploration missions. First of all, unlike lunar missions, asteroids are far away from the earth. In order to explore asteroids at close range, deep-space probes need to travel long distances in harsh conditions. Second, the landing duration of the probe is relatively short, so the topographic map of the landing area should be obtained by using $3 \mathrm{D}$ reconstruction technology before landing. Moreover, the small size, irregular shape and uneven mass distribution of asteroids further increase the difficulty of landing missions.

Most of today's space probes are based on their onboard sensors, such as Light Detection and Ranging (LiDAR), camera, laser altimeter, etc., for navigation and topographic mapping. Some researchers proposed a method of navigation based on LiDAR for landing. They simulated the landing process of the Mars rover. And based on the DEM generated by LiDAR data, they gave the choice of landing site (Johnson et al. 2002). The Hayabusa probe, designed by Japan Aerospace Exploration Agency (JAXA), used altitude information from a laser altimeter to map the topography of Itokawa (Yano et al. 2006). Some researchers of European Space Agency (ESA) proposed an autonomous navigation algorithm using laser ranging and microwave measurements to solve the problem of autonomous navigation and control of the landing in the Rosetta mission. In general, these active measurement methods are robust to light background. But due to the measurement range and the measurement errors, their application scenarios are also limited.

In addition, passive image-based terrain mapping and positioning methods have been developed. These methods obtain terrain information from images of the asteroid's surface taken by mounted cameras. Kawaguchi et al. (1999) used the spacecraft's navigation camera to take pictures of the asteroid's surface and then extracted feature points from the images for autonomous positioning. Johnson and Matthies (1999) proposed a method to estimate the motion parameters of soft landing by using computer vision and image processing technology. This method used the image sequence collected at the landing stage to recover the 3D motion parameters and structural parameters from the 2D image. China's Chang'E-3 and Chang'e-4 probes also used optical navigation by using camera and LIDAR data to support the soft landing. In the Hayabusa2 mission, researchers used decent images to generate the DEM around the landing area. During the sampling process, Hayabusa2 had to land precisely on a preselected flat surface to avoid potential hazards. Compared with the active measurement method, the image-based

* Corresponding author 
topographic mapping method is more limited by illumination condition, but its measurement range can be larger than that of active measurement method. Besides, the camera is lighter than the LiDAR, making it easier for the spacecraft to carry.

Due to the difficulties and high cost of the asteroid exploration mission, the whole process need to be verified by simulation in advance. Therefore, simulation and topographic mapping are crucial in preparation of asteroid exploration missions. In this paper, we presented a topographic mapping and analysis method based on 3D reconstruction model of a simulated asteroid. The rest of the paper is organized as follows. Section 2 describes the proposed method in three parts. In Section 3, experiments are reported to analyse the performance of our method. Conclusions and future works are discussed in the last section.

\section{METHODOLOGY}

In this section, the proposed topographic mapping method consists of three major components. First, in order to generate the DEM and DOM required for selection of the landing/sampling area, a 3D reconstruction of a simulated asteroid was carried out using SfM technique. After that, the 3D point cloud data was used to generate DEM and DOM. Finally, by analysing the DEM data of asteroid, the obstacle distribution map was generated. Detailed processes in each components are given in the following subsections.

\subsection{D Reconstruction}

Figure 1 shows the schematic of 3D reconstruction. The grey sphere represents the simulated asteroid we used, with a diameter of about 1 meter. It is connected to an electric base, which can rotate simulated asteroid at a certain speed. The blue and green objects represent the camera and the laser rangefinder, respectively. We first used the camera to obtain images of the simulated asteroid. By rotating the electric base, we took images from different directions with sufficient overlap, enabling a more complete coverage.
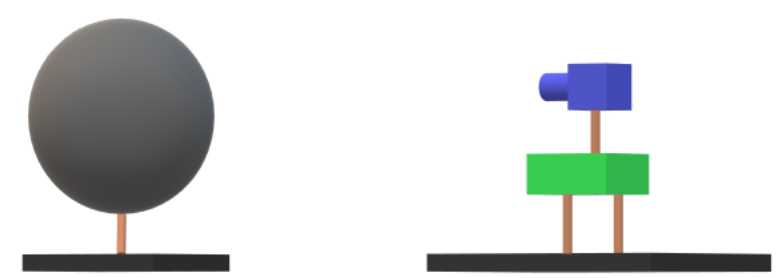

Figure 1. The schematic of the equipment used in our research

Then, these images were taken as the input data to compute correspondences, i.e., image matching. Figure 2 shows the flow of this part. We detected features in each image by using SIFT algorithm (Lowe, 2004) and matched keypoints between each pair of images. For each pair, we estimate a fundamental matrix and refine matches by using Random Sample Consensus (RANSAC) algorithm (Nister, 2008). After all pairwise matches are determined, we input correspondences into SfM part.

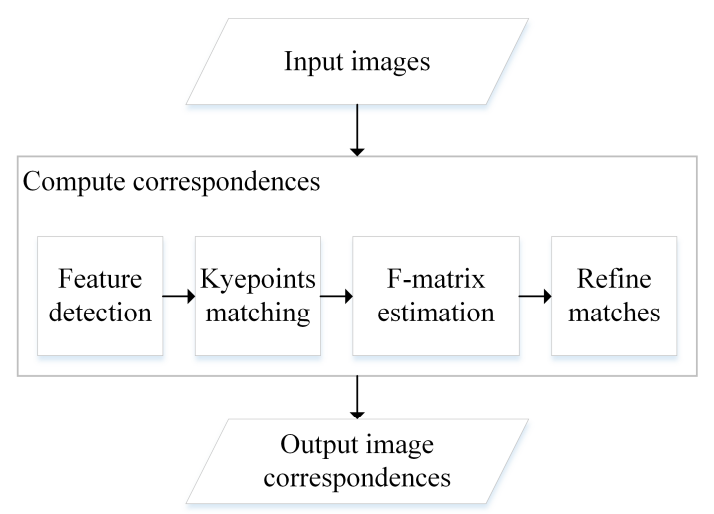

Figure 2. The process of computing image correspondences

As Figure 3 shows, we first selected good image pair to derive an initial reconstruction. This image pair should have enough matching points. The five-Point algorithm (Nister, 2004) was used to estimate the extrinsic parameters of the matching pair, and the 3D points of initial model can be obtained after the triangulation process. Then we iteratively added new images and calculated its $3 \mathrm{D}$ points. The newly obtained $3 \mathrm{D}$ points were fused with the previously calculated $3 \mathrm{D}$ points. Through the multi-view projective SfM process, the extrinsic parameters of cameras are obtained and the $3 \mathrm{D}$ point cloud of the simulated asteroid was reconstructed.

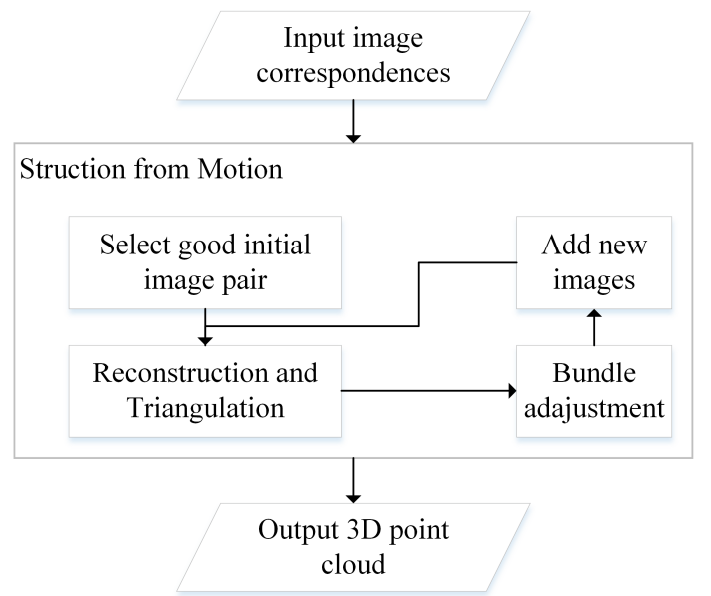

Figure 3. The process of SfM

The 3D point cloud data at this time were in model coordinates without an absolute scale. Therefore, we used a laser rangefinder to establish a coordinate system with respect to the laser emission centre as the origin, so as to convert the model coordinates to the world coordinates. Finally, the origin of world coordinates was shifted to the geometrical centre of the point cloud data through coordinate transformation.

\subsection{Topographic Mapping}

Through the $3 \mathrm{D}$ reconstruction process, we obtained the point cloud data of the simulated asteroid. Then we converted the coordinates of these 3D points to the coordinate of longitude and latitude. After that, Delaunay Triangulated Irregular Network (TIN) was generated using point by point inserting method. And an interpolation method was adopted to build regular grid DEM at specified resolution. For each grid on DEM, we adopted the colour information contained in the image near the zenith of the grid, so as to generate the DOM. 


\subsection{Generation of Obstacle Distribution Map}

For the successful implementation of the asteroid exploration mission, it is necessary to select suitable and safe landing/sampling areas for the lander. So we calculated the slope and elevation step values of each grid of the DEM. In order to calculate these two values, we first set up a calculation window for each grid. Then we fit a plane equation according to the elevation values of the grids in the window. The plane equation is shown in Equation (1).

$$
A x+B y+C z+D=0
$$

Based on the normal vector of the plane, we calculated the slope value of each grid through Equation (2). And the slope map is obtained by calculating the corresponding slope value of all grids. The larger the slope value, the steeper the terrain.

$$
\beta=\arctan \sqrt{\frac{A^{2}+B^{2}}{C^{2}}}
$$

where $\beta=$ slope value

$$
A, B, C=\text { normal vector of the plane equation }
$$

As for elevation step value, it is the difference in elevation within the calculation window. As shown in Equation (3), it is calculate as the difference between the maximum elevation minus the minimum elevation, The elevation step value reflects the intensity of elevation change within a certain region. The bigger the elevation step is, the greater the elevation drop in the region is. Therefore, it is an important indicator to ensure the safe landing.

$$
H_{\text {diff }}=H_{\max }-H_{\min }
$$

where $H_{\text {diff }}$ is elevation step value, $H_{\max }$ is maximum elevation value in the window, and $H_{\min }=$ min elevation value in the window.

After computing the slope and elevation step values, we compared them with the pre-set thresholds, if both the two values of a grid are smaller than the respective thresholds, the grid was marked as non-obstacle. Otherwise, the grid was marked as an obstacle. Finally, according to the labelled obstacle grids, the obstacle distribution map was generated.

\section{EXPERIMENTAL RESULTS}

In this part, we used an equipment shown in Figure 1 to verify our method. Before starting the 3D reconstruction experiment, we calibrated the intrinsic parameters of the camera. As Figure 4 shows, we took photos of the calibration plate from different distances and different angles. We used a MATLAB-based calibration toolbox (Zhang, 2000) to calculate the calibration parameters of the RGB camera. And the results shown in Table 1. The unit of $f_{x}, f_{y}, c_{x}$, and $c_{y}$ is pixel.

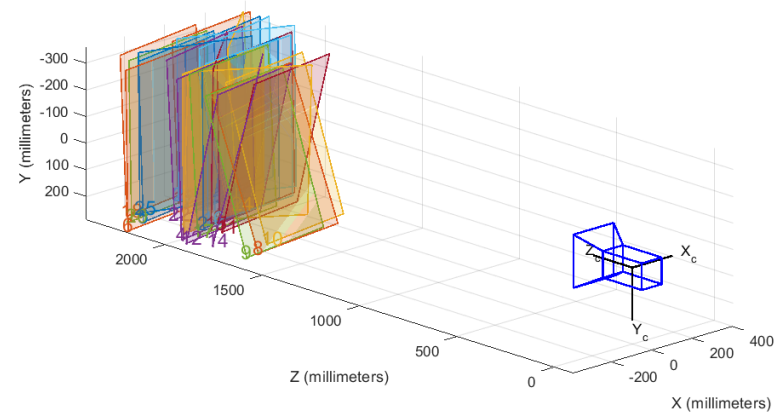

Figure 4. Diagram of camera calibration

\begin{tabular}{|c|c|c|c|}
\hline \multicolumn{2}{|c|}{ Focal length } & \multicolumn{2}{c|}{ Principal point } \\
\hline$f_{x}$ & $f_{y}$ & $c_{x}$ & $c_{y}$ \\
\hline 4726.03 & 4723.00 & 1043.20 & 1009.05 \\
\hline
\end{tabular}

Table 1 . The intrinsic parameters of the camera

Then an electric base was used to rotate the simulated asteroid at a slow speed, while using the camera to capture images from multiple orientations as Figure 5 shows. We obtained 659 images with a size of $2048 \times 2048$ pixels. Figure 6 shows some captured images.

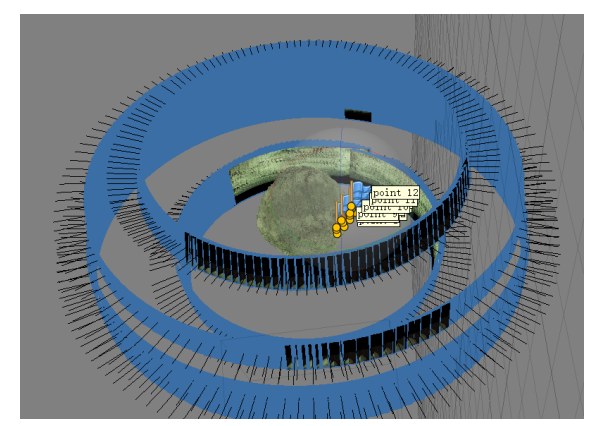

Figure 5. Captured images from multiple orientations
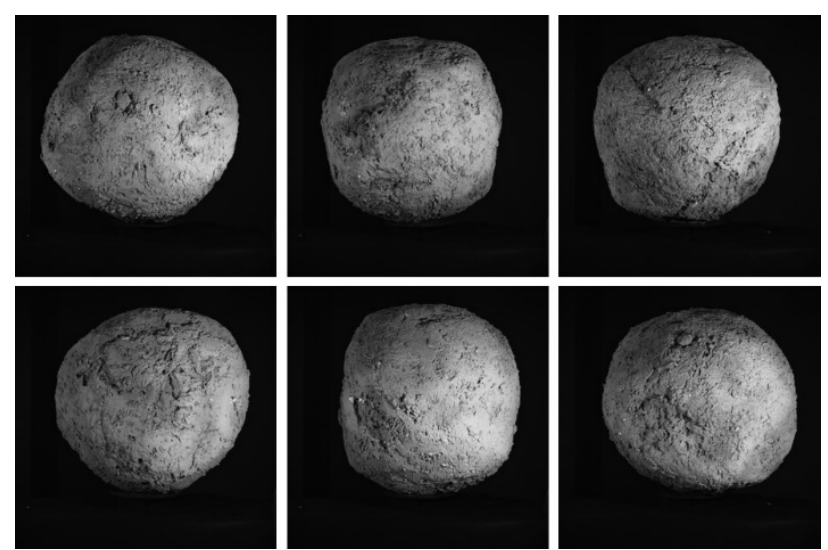

Figure 6. Example images captured by the camera

Based on these images, we completed the $3 \mathrm{D}$ reconstruction of the simulated asteroid through the processes shown in Figure 2 and Figure 3, resulting in the 3D point cloud of simulated asteroid. Figure 7 shows the dense point cloud and the textured model of $3 \mathrm{D}$ reconstruction results. 

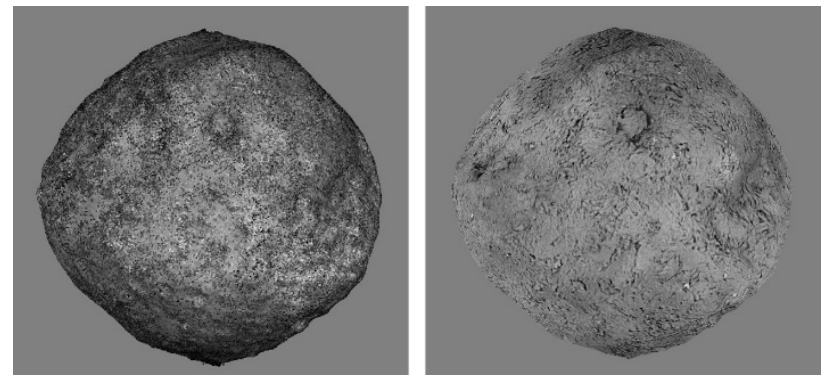

Figure 7. Dense point cloud and textured 3D model

Here the 3D point cloud data were still in model coordinates without an absolute scale information. We established a coordinate system with the laser emission centre as the origin, so as to convert the model coordinates to the absolute coordinates. Figure 8 shows the distribution of control points and check points. Tables 2 and 3 list the coordinates of control points and check points, respectively.

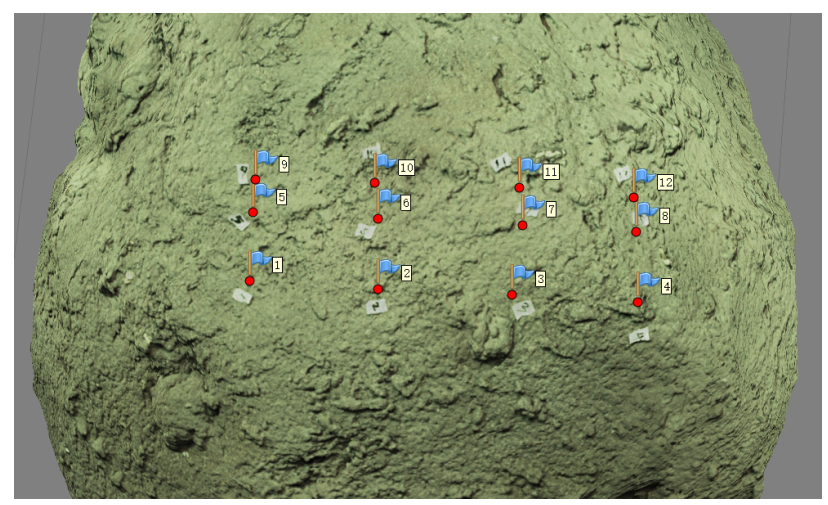

Figure 8 . The control points and check points.

\begin{tabular}{|c|c|c|c|}
\hline Control points & $\mathrm{X}(\mathrm{m})$ & $\mathrm{Y}(\mathrm{m})$ & $\mathrm{Z}(\mathrm{m})$ \\
\hline 1 & 0.000 & 0.000 & 1.085 \\
\hline 4 & 0.300 & 0.000 & 0.924 \\
\hline 7 & 0.200 & -0.060 & 0.961 \\
\hline 9 & 0.000 & -0.090 & 1.095 \\
\hline 12 & 0.300 & -0.090 & 0.948 \\
\hline
\end{tabular}

Table 2. The coordinates of the control points

\begin{tabular}{|c|c|c|c|}
\hline Check points & $\mathrm{X}(\mathrm{m})$ & $\mathrm{Y}(\mathrm{m})$ & $\mathrm{Z}(\mathrm{m})$ \\
\hline 2 & 0.100 & 0.000 & 1.015 \\
\hline 3 & 0.200 & 0.000 & 0.966 \\
\hline 5 & 0.000 & -0.060 & 1.091 \\
\hline 6 & 0.100 & -0.060 & 1.034 \\
\hline 8 & 0.300 & -0.060 & 0.939 \\
\hline 10 & 0.100 & -0.090 & 1.048 \\
\hline 11 & 0.200 & -0.090 & 0.964 \\
\hline
\end{tabular}

Table 3. The coordinates of the check points

Then we shifted the origin of absolute coordinates to the geometrical centre of 3D point cloud through coordinate transformation. And we calculated the errors of the control points and check points, as shown in Table 4 and Table 5. It can be seen from the small errors of the control points and check points that the millimetre-level accuracy of $3 \mathrm{D}$ point cloud meets the requirements of topographic mapping.

\begin{tabular}{|c|c|}
\hline \multicolumn{2}{|c|}{ Control points } \\
\hline Number & Error(m) \\
\hline 1 & 0.002164 \\
\hline 4 & 0.003406 \\
\hline 7 & 0.003633 \\
\hline 9 & 0.001191 \\
\hline 12 & 0.000950 \\
\hline
\end{tabular}

Table 4. The errors of the control points

\begin{tabular}{|c|c|}
\hline \multicolumn{2}{|c|}{ Check points } \\
\hline Number & Error(m) \\
\hline 2 & 0.005347 \\
\hline 3 & 0.001728 \\
\hline 5 & 0.000976 \\
\hline 6 & 0.002622 \\
\hline 8 & 0.002514 \\
\hline 10 & 0.003355 \\
\hline 11 & 0.002879 \\
\hline
\end{tabular}

Table 5 . The errors of the check points

After that, based on 3D point cloud, we generated DEM and DOM using interpolation algorithm. Figure 9 shows the DEM between latitudes of south and north 70 degree. A change in colour represents a change in elevation value. The redder the colour, the higher the elevation. And the corresponding DOM is shown in Figure 10. By combining DEM and DOM, we can see the elevation changes of terrain intuitively.

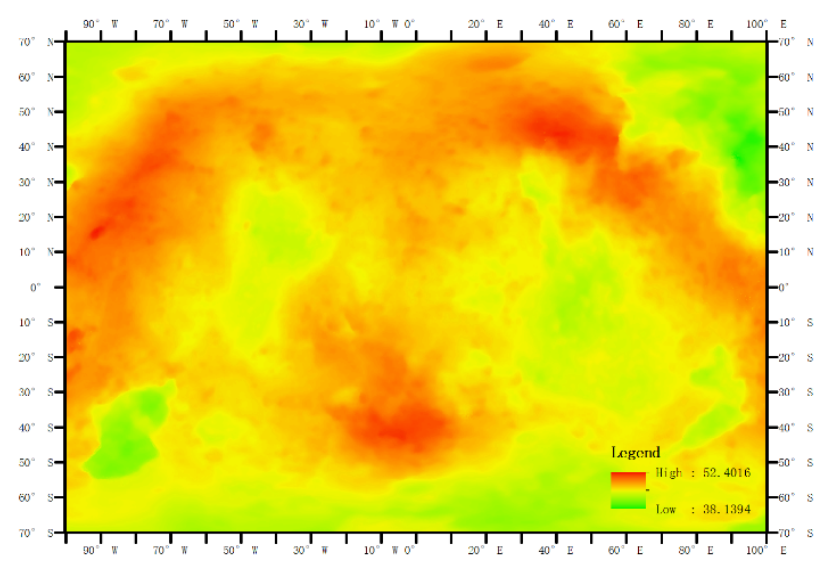

Figure 9. DEM between latitudes of $70^{\circ} \mathrm{N}$ and $70^{\circ} \mathrm{S}$

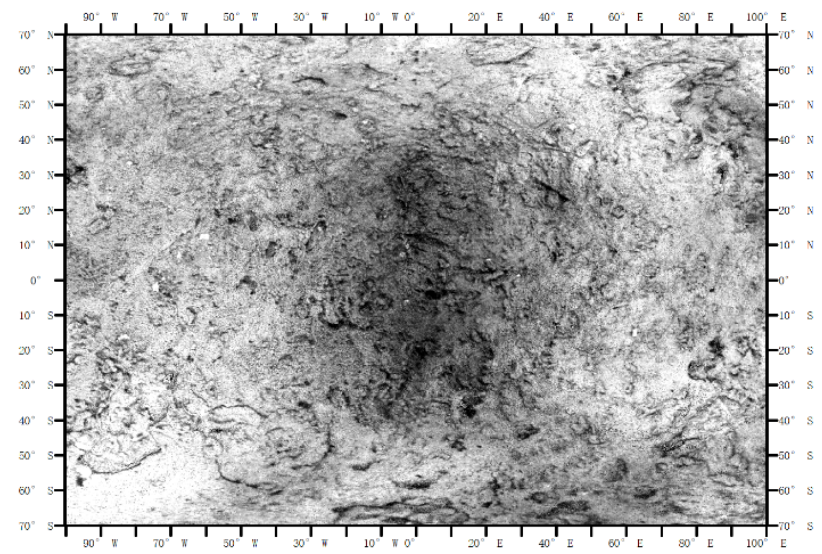

Figure 10. DOM between latitudes of $70^{\circ} \mathrm{N}$ and $70^{\circ} \mathrm{S}$ 
For topographic analysis, we calculated the slope and elevation step values using the DEM. Figure 11 shows the slope results and Figure 12 shows the elevation step values. As can be seen from them, the two values increase at the edge of some simulated craters.

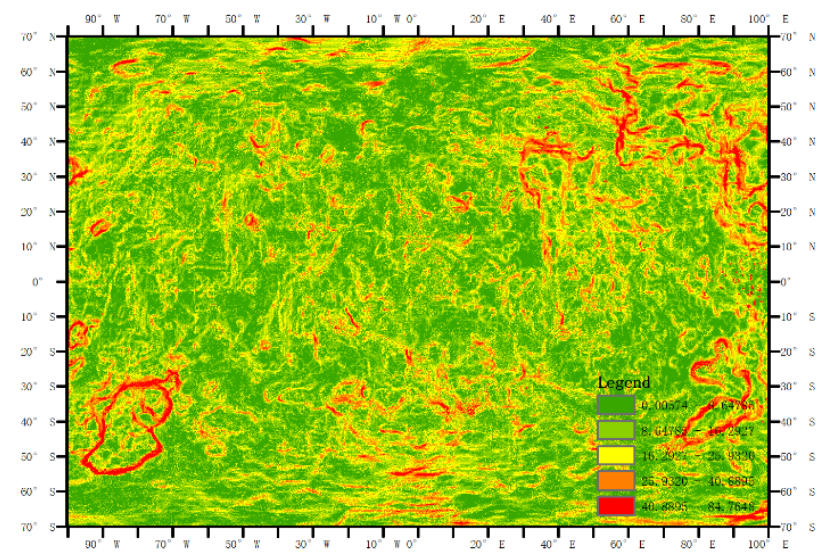

Figure 11. Slope map of the simulated asteroid

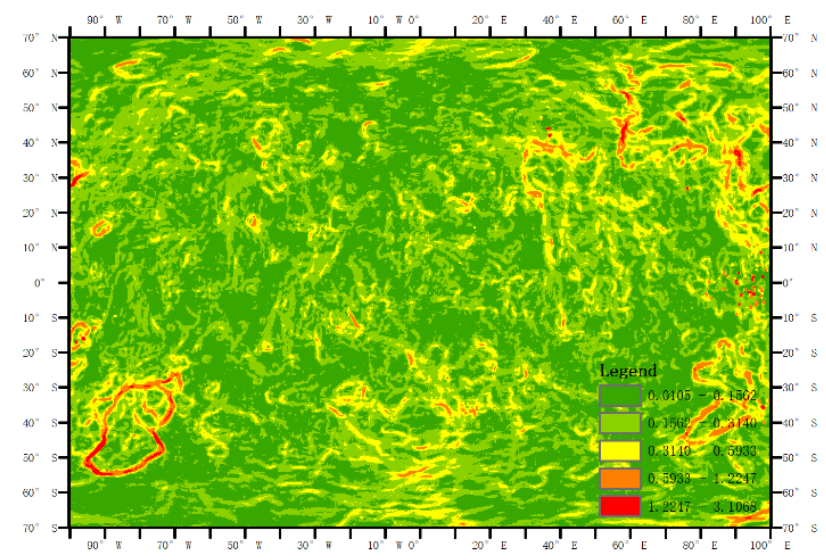

Figure 12. Elevation step of the simulated asteroid

The slope threshold was set at 15 degrees and the threshold of the elevation step was set at 0.3 meters. Then we compared slope results and elevation step results with the two thresholds. As a result, we obtained the pixels labelled as obstacle and nonobstacle. Finally, according to the distribution of labelled pixels, we generated the obstacles map as Figure 13 shows. In Figure 13, red colour represents obstacle and green colour represents nonobstacle. Based on the calculated obstacle distribution map, we can assess the landing/sampling area of the lander.

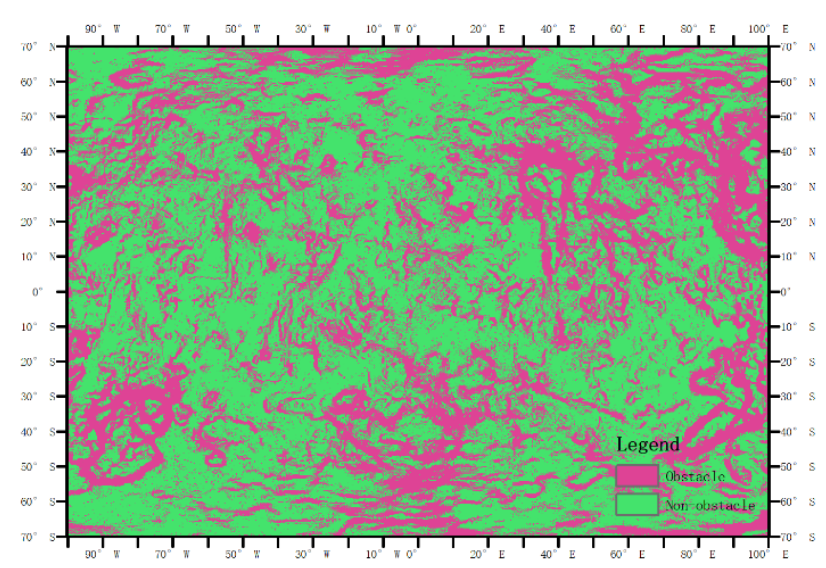

Figure 13. Obstacle distribution map the simulated asteroid

\section{CONCLUSION}

In this paper, we presented a topographic mapping and analysis method based on 3D reconstruction model of a simulated asteroid. First, 3D reconstruction of a simulated asteroid was achieved using SfM technique. Then DEM and DOM were produced based on the 3D point cloud data. By analysing the DEM data, we calculated the slope and elevation step values. Based on these two results and pre-set thresholds we finally obtained the obstacles distribution map.

In the experiment section, we used a simulated asteroid to conduct experiments and evaluation. All the experimental results show that the proposed method is able to reconstruct a $3 \mathrm{D}$ point cloud of simulated asteroid and conduct topographic mapping with a millimetre accuracy, demonstrating the effectiveness of the developed method.

The topographic mapping and analysis method presented in this study can be applied to landing/sampling area selection in further asteroid exploration research. Our next work is to verify and improve our method by introducing illumination changes in the experiments.

\section{ACKNOWLEDGEMENTS}

This research was supported by the Strategic Priority Research Program of the Chinese Academy of Sciences (Grant No. XDA1502030503).

\section{REFERENCES}

Johnson, A. E., Klumpp, A. R., Collier, J. B., Wolf, A. A., 2002. Lidar-based hazard avoidance for safe landing on mars. Journal of Guidance Control \& Dynamics 25(6), 1091-1099.

Johnson, A. E., Matthies, 1999. Precise image-based motion estimation for autonomous smallbody exploration. In: Proc.of the 5th i-SAIRAS, Noordwijk, The Netherlands.

Johnson, A. E., Montgomery, J. F., 2008. Overview of terrainrelative navigation approaches for precise lunar landing. In: Proc. of the IEEE Aerospace Conference, Big Sky, Montana.

Kawaguchi, J., Hashimoto, T., Misu T., Sawai, S., 1999. An Autonomous Optical Guidance and Navigation around the 
Asteroid. In: Proc.of the 47th International Astronautical Congress, Beijing, China.

Lowe, D. G., 2004: Distinctive image features from scaleinvariant keypoints. International Journal of Computer Vision 60(2), 91-110.

Nister, D., 2004: An efficient solution to the five-point relative pose problem. IEEE Transactions on Pattern Analysis and Machine Intelligence 26(6), 756-770.

Nister, D., 2008. Preemptive RANSAC for Live Structure and Motion Estimation. In: Proc.of the 9th IEEE International Conference on Computer Vision, Nice, France.

Wu, W. R., Dong, G. L., Li, H. T., 2013: Engineering and Technology of Deep Space TT\&C System. Science Press, Beijing.

Yano, H., Kubota, T., Miyamoto, H., Okada, T., Scheeres, D., Takagi, Y., Yoshida, K., Abe, M., Abe, S., Barnouin-Jha, O., Fujiwara, A., Hasegawa, S., Hashimoto, T., Ishiguro, M., Kato, M., Kawaguchi, J., Mukai, T., Saito, J., Sasaki, S., Yoshikawa, M., 2006: Touchdown of the Hayabusa Spacecraft at the Muses Sea on Itokawa. Science 312(5778), 1350-1353.

Zhang, Z., 2000: A flexible new technique for camera calibration. IEEE Transactions on Pattern Analysis and Machine Intelligence 22(11), 1330-1334. 